\title{
Deafferented controllers: a fundamental failure mechanism in cortical neuroprosthetic systems
}

\author{
Ferran Galán* and Stuart N. Baker \\ Movement Laboratory, Institute of Neuroscience, Newcastle University, Newcastle upon Tyne, UK
}

Brain-machine interface (BMI) research assumes that patients with disconnected neural pathways could naturally control a prosthetic device by volitionally modulating sensorimotor cortical activity usually responsible for movement coordination. However, computational approaches to motor control challenge this view. This article examines the predictions of optimal feedback control (OFC) theory on the effects that loss of motor output and sensory feedback have on the normal generation of motor commands. Example simulations of unimpaired, totally disconnected, and deafferented controllers illustrate that by neglecting the dynamic interplay between motor commands, state estimation, feedback and behavior, current BMI systems face translational challenges

OPEN ACCESS

Edited by:

George W. Huntley,

Icahn School of Medicine at Mount

Sinai, USA

Reviewed by:

Joshua C. Brumberg,

City University of New York, USA

Quentin Barraud,

École Polytechnique Fédérale de

Lausanne, Switzerland

*Correspondence:

Ferran Galán,

The Medical School, Institute of

Neuroscience, Newcastle University,

Henry Wellcome Building, Framlington

Place, Newcastle upon Tyne,

NE2 4HH, UK

ferran.galan@ncl.ac.uk

Received: 20 April 2015

Accepted: 03 July 2015

Published: 17 July 2015

Citation:

Galán F and Baker SN (2015)

Deafferented controllers: a fundamental failure mechanism in cortical neuroprosthetic systems.

Front. Behav. Neurosci. 9:186.

doi: 10.3389/fnbeh.2015.00186 rooted in a debatable assumption and experimental models of limited validity.

Keywords: brain-machine interface (BMI), optimal feedback control, dynamical systems, motor control, neuroprosthetics

\section{Introduction}

Current BMI research (Bensmaia and Miller, 2014) aims at extracting movement parameters believed to be encoded in motor cortical areas that would enable neuroprosthetic control after peripheral disconnection. However, despite encouraging early laboratory monkey experiments (Taylor et al., 2002; Carmena et al., 2003; Moritz et al., 2008; Velliste et al., 2008; Ethier et al., 2012), cortical neuroprosthetic control (Hochberg et al., 2012; Collinger et al., 2013) is poor compared to natural movements and highly dependent on visual feedback. Understanding the mechanisms underlying poor BMI performance is the key to clinical translation; currently funded efforts focus on identifying failure mechanisms associated with degraded neural signal acquisition from chronically implanted electrode arrays, unreliable motor decoding of such neural signals, and delivery of poor sensory feedback (Miranda et al., 2015). This article considers a fundamental failure mechanism largely underappreciated by the BMI community: the assumption that sensorimotor cortical activity engaged during unimpaired movement remains available for prosthetic control after peripheral disconnection.

Especially relevant to BMI research, the OFC theory of motor coordination (Todorov and Jordan, 2002) highlights the dynamic interplay between motor commands and sensory feedback, discouraging ideas of neural coding/decoding and emphasizing neural dynamics when interpreting sensorimotor function (Scott, 2012; for a recent review of OFC and its possible biological underpinnings see Scott et al., 2015; Shenoy et al., 2013). This is supported by work demonstrating the influence of sensory feedback on ongoing M1 activity (Herter et al., 2009; Suminski et al., 2010; Pruszynski et al., 2011), studies describing changes in neural tuning at different time scales (Sergio et al., 2005; Hatsopoulos et al., 2007; Churchland et al., 2012) 
and under different feedback conditions (Gaunt et al., 2013), and work showing altered EEG movement-related information in the absence of kinaesthetic feedback (Galán et al., 2015).

The OFC framework further predicts that loss of motor output and sensory feedback will have an impact on the normal generation of motor commands; although these might still be initiated, they are likely to be altered compared with those responsible for producing unimpaired movement. Such a prediction poses relevant questions to BMI research that need addressing: is it possible at all after peripheral disconnection to use sensorimotor cortical activity for reliable cortical neuroprosthetic control? If not, what is needed? And, in such a scenario, how can studies with able-bodied experimental subjects contribute to advancing the development of reliable BMI systems? The following sections illustrate the predictions of an infinite-horizon OFC model (Qian et al., 2013) in situations with different types of peripheral disconnection, and discuss the implications for the BMI field.

\section{Peripheral Disconnections in OFC}

Figure 1 illustrates the OFC framework. To achieve a goal such as an arm movement, optimal feedback controllers generate motor commands that minimize a cost function (which might include error and energy expenditure) based on their belief about the current state of the arm and the world. Such a belief is formed by integrating through an optimal estimator the state changes observed through delayed afferent feedback with those predicted by forward models, which use knowledge about the system dynamics acting on a copy of the motor commands. Note that motor commands and sensory feedback are both susceptible to corruption by noise. References (Shadmehr and Krakauer, 2008; Scott, 2012; Shadmehr and Mussa-Ivaldi, 2012; Scott et al., 2015) provide further detail on the computational and neural basis of the OFC framework in voluntary motor control.

Understanding the causal links between different movement phases such as preparation and execution is especially relevant to cortical neuroprosthetic control (Shenoy et al., 2011). An interesting property of the infinite-horizon OFC model utilized here (Qian et al., 2013) is the integration of movement control and postural maintenance, without artificial separations. This occurs through the application of steady-state solutions for state estimation, movement execution and postural maintenance. The state estimator and controller are computed in a preparatory phase before movement starts, but motor commands can only be determined during the movement attempt as they depend on estimated states and sensory feedback. Therefore, movement properties such as duration and trajectory, as well as the motor commands themselves, emerge from the model with inherent trial-to-trial variability. In brief, Qian et al. (2013) consider a linear dynamical system governed by stochastic differential equations:

$$
\begin{aligned}
& d x=(A x+\varphi B u) d t+F x d \beta+Y u d \gamma+G d \omega, \\
& d y=C x d t+D d \xi
\end{aligned}
$$

where $x$ is the state $n$-vector, $u$ is the control $k$-vector (motor command), and $y$ is the sensory feedback vector. $\beta$ and $\gamma$ are scalar Wiener processes, and $\omega$ and $\xi$ are $n$ - and $k$ vector Wiener processes modeling noise in control and sensory

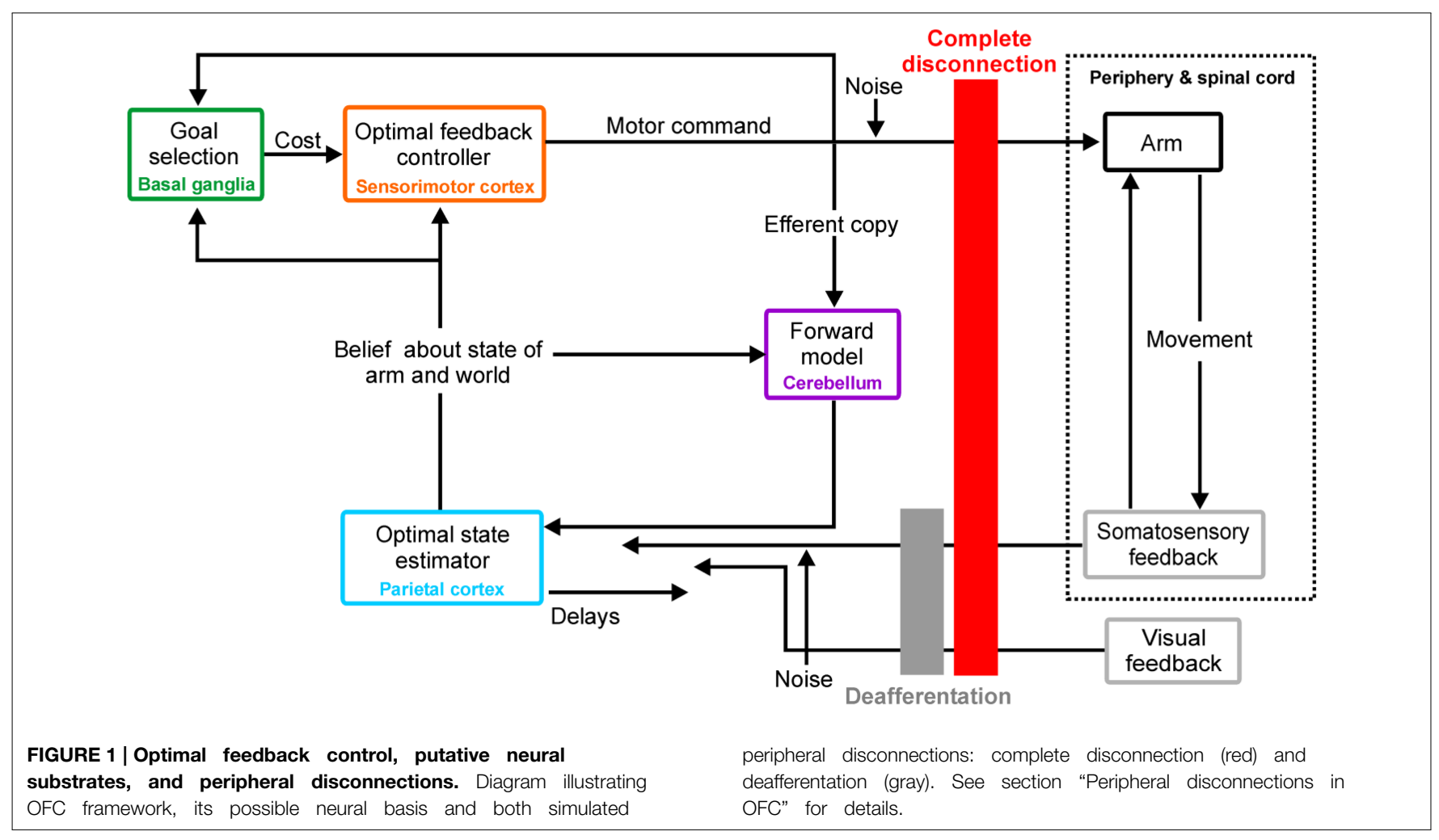


feedback, all independent from each other and standardized so that over a time step $d t$, the corresponding Gaussian white noise processes have variance $d t . A, B, C, D, F, G$ and $Y$ are constant matrices; $A$ and $B$ define the motor plant according to Newtonian mechanics and the muscle force equation, $C$ and $D$ define observation and observation noise, $F$ and $Y$ define stateand control-dependent noise (multiplicative or signal dependent noise), and $G$ defines control-independent noise. $\varphi$ is a scalar modeling efferent disconnection: if the system remains intact then $\varphi=1$, if otherwise motor commands are blocked then $\varphi=0$. The current state $x$ is not available for control and is estimated according to a linear Kalman filter (Kalman, 1960):

$$
d \hat{x}=(A \hat{x}+B u) d t+K(d y-C \hat{x} d t),
$$

where the first term is the prediction based on a forward internal model and an efference copy of the control signal, and the second term is the correction based on the discrepancy between observed and predicted sensory feedback.

The motor command is a linear function of $\widehat{x}$ :

$$
u=-L \hat{x},
$$

and estimator gain $K$ and controller $L$ are determined by optimizing costs. According to Phillis's infinite-horizon formulation (Phillis, 1985), the estimator cost is defined as the steady-state variance of the estimation error $\tilde{x} \equiv x-\hat{x}$,

$$
J_{1}=\lim _{t \rightarrow \infty} E\left[\tilde{x}^{T} U \tilde{x}\right],
$$

and the controller cost as the steady-state cost per unit of time,

$$
J_{2}=E\left[\lim _{t \rightarrow \infty} \frac{1}{t} \int_{0}^{t}\left(x^{T} Q x+u^{T} R u\right) d t\right],
$$

where the first term measures accuracy of the reaching target, the second term measures energetic cost, and matrices $U, Q$, and $R$ are constant and symmetric.

The simulations reproduced unimpaired single-joint reaching movements of the forearm at the elbow as in (Qian et al., 2013), but additionally tested two different types of peripheral disconnection (see Figure 1): complete disconnection (disconnected, $K=\varphi=0$ ) and deafferentation (deafferented, $K=0$ ). First, estimator gain $K$ and controller $L$ were numerically obtained from an unimpaired controller as in (Qian et al., 2013); note that they only depend on plant and cost parameters. Then, optimal steady state estimator gain $K$ and controller $L$ were applied to move the hand $(n=20$ trials) toward a target placed $50 \mathrm{~cm}$ away according to the system dynamics defined by unimpaired, disconnected, and deafferented conditions. (Matlab code obtained from N. Qian upon request and modified to simulate deafferentation and complete disconnection).

Figure 2 shows time-resolved profiles of hand position, motor command, and their variance with respect to the average, generated by the system dynamics defined by unimpaired, disconnected, and deafferented controllers. The model reproduced typically-observed properties of unimpaired movements with trial-to-trial variability (Qian et al., 2013) (Figures 2A-D, unimpaired). By contrast, the disconnected controller generated (Equation 4) highly stereotyped (Figures 2B,D; disconnected) motor commands based on estimates that solely rely on the predictions of a forward model using efference copy (Wolpert and Miall, 1996; Wolpert et al., 1998) (note that $K=0$ in Equation 3). Such motor commands generated no overt output, however, due to the efferent block (Figures 2A,C; disconnected).

These results seem to suggest that not only is the motor command intact in the disconnected state, but it is actually less variable than in the unimpaired condition. Does this mean that decoding of the motor command would lead to effective movements of a neural prosthetic? Such decoding can be modeled using a deafferented controller, where efferents remain connected, revealing what movements would be generated from the stereotyped command seen in the disconnected state. This revealed highly variable movement trajectories, resulting from the accumulation of errors over time in the state estimate which must rely, in the absence of sensory feedback, on only forward model predictions from efference copy. The monotonic increase of state variance reproduced movement profiles displayed by patients suffering from sensory neuropathies without visual feedback (Sanes et al., 1984; Gordon et al., 1995) (Figures 2A,C; deafferented). Paradoxically, the generation of motor commands with trial-to-trial variability ensures successful movements (Todorov and Jordan, 2002) (Figure 2 unimpaired), whereas the lack of such variability ensures movement imprecision (Figure 2 deafferented). These examples illustrate that the lack of sensory feedback from the periphery suffered by disconnected and deafferented controllers has an impact on the generation of motor commands via poor state estimation. Execution of such motor commands (e.g., by a decoder driving a neural prosthetic) unavoidably leads to poor motor control.

\section{Discussion}

Current BMI systems aim to extract neural information from sensorimotor cortical areas to enable prosthetic control to the paralyzed. However, the above examples illustrate that these areas, considered as optimal feedback controllers (see Figure 1; Shadmehr and Krakauer, 2008; Scott, 2012), are likely to generate motor commands leading to poor prosthetic control when disconnected from the periphery. An ideal bidirectional biomimetic approach which restored both sensory and motor function would theoretically allow for feedback-corrected state estimation and restore reliable control; recent work on hand prosthetics enabling effective modulation of grasping force by conveying nearly "natural" sensory feedback (Raspopovic et al., 2014) supports this notion. However, such interfacing at higher levels of the neuraxis is beyond current technical developments (Bensmaia and Miller, 2014). More feasible might be an implementation of bidirectional interfacing based on learned non-biomimetic associations (Bensmaia and Miller, 2014). Studies with able-bodied experimental subjects demonstrating volitional control of sensorimotor cortical activity (Fetz, 1969), 

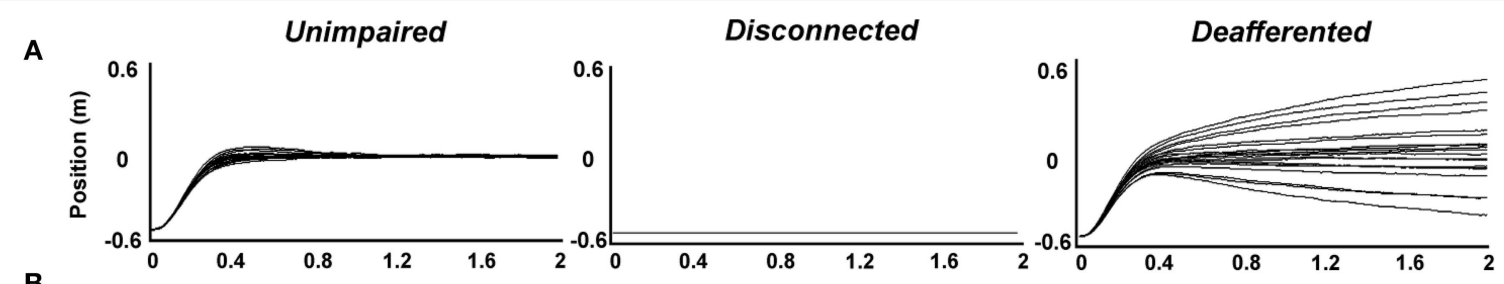

B
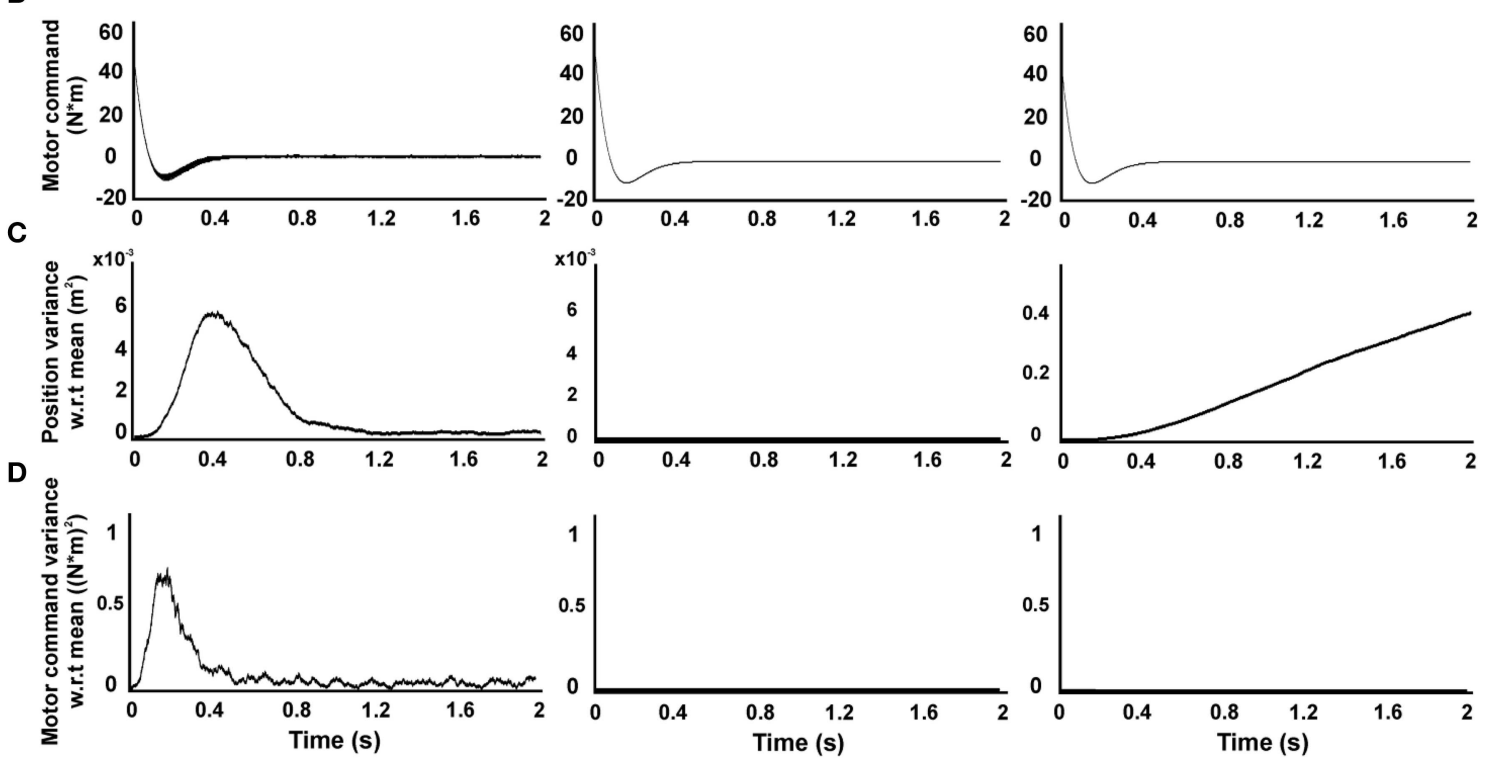

FIGURE 2 | Simulations of reaching movements produced by unimpaired, disconnected, and deafferented controllers. Simulated single-joint reaching movements of the forearm $(n=20)$ toward a target placed $50 \mathrm{~cm}$ from starting position. (A) Position (B) motor command (C) position variance with respect to the average. (D) Motor command variance with respect to the average. neural adaptation during efferent BMI control (Jarosiewicz et al., 2008; Ganguly and Carmena, 2009) and discrimination of evoked percepts by intracortical microstimulation (ICMS) to somatosensory cortex (Romo et al., 1998; London et al., 2008; O'Doherty et al., 2011) all support that this approach may have merit, although it remains unknown whether this will deliver sufficiently rich sensory feedback to enable precise cortical neuroprosthetic control. At present, deafferented patients who only rely on visual feedback appear to provide an upper bound on current cortical neuroprosthetic systems' performance.

In Figure 1 we have assigned putative neural structures to each component of the OFC model. In reality the system is likely to be distributed, with a single box in that diagram implemented by multiple neural circuits at different levels of the neuraxis. Feedback is also distributed, being received for example by both spinal and cortical circuits. One consequence of such an arrangement may be that the full motor command can never be read out from one neural structure on its own.

How do the ideas presented above fit with experimental results from the BMI field? Two FDA clinical trials involving patients with paralysis (Hochberg et al., 2012; Collinger et al., 2013) showed impoverished cortical control compared to natural movements, in agreement with these concepts. Other than these reports, the majority of BMI work involve able-bodied non-human primates (Shenoy and Carmena, 2014), with intact sensory feedback loops from the periphery. In just a few instances was peripheral disconnection modeled with nerve blocks at upper arm (Moritz et al., 2008) and elbow (Pohlmeyer et al., 2009; Ethier et al., 2012), but even in those cases feedback above the block was intact. The ability to use residual sensorimotor loops has been previously demonstrated in amputees (Kuiken et al., 2007; Raspopovic et al., 2014), where access to motor representations of the missing limb appears conditional upon the re-establishment of peripheral connections (Reilly et al., 2006). Evidence for effective neuroprosthetic systems based on extracting control signals from sensorimotor cortical areas totally disconnected from the periphery is currently lacking. We argue that neuroprostheses which seek to exploit preserved sensorimotor loops may offer the most promising control alternatives on theoretical grounds.

\section{Acknowledgments}

This work was supported by the Wellcome Trust. 


\section{References}

Bensmaia, S. J., and Miller, L. E. (2014). Restoring sensorimotor function through intracortical interfaces: progress and looming challenges. Nat. Rev. Neurosci. 15, 313-325. doi: $10.1038 / \mathrm{nrn} 3724$

Carmena, J. M., Lebedev, M. A., Crist, R. E., O’Doherty, J. E., Santucci, D. M., Dimitrov, D. F., et al. (2003). Learning to control a brain-machine interface for reaching and grasping by primates. PLoS Biol. 1:E42. doi: 10.1371/journal.pbio.0000042

Churchland, M. M., Cunningham, J. P., Kaufman, M. T., Foster, J. D., Nuyujukian, P., Ryu, S. I., et al. (2012). Neural population dynamics during reaching. Nature 487, 51-56. doi: 10.1038/nature11129

Collinger, J. L., Wodlinger, B., Downey, J. E., Wang, W., Tyler-Kabara, E. C., Weber, D. J., et al. (2013). High-performance neuroprosthetic control by an individual with tetraplegia. Lancet 381, 557-564. doi: 10.1016/S01406736(12)61816-9

Ethier, C., Oby, E. R., Bauman, M. J., and Miller, L. E. (2012). Restoration of grasp following paralysis through brain-controlled stimulation of muscles. Nature 485, 368-371. doi: 10.1038/nature10987

Fetz, E. E. (1969). Operant conditioning of cortical unit activity. Science 163, 955-958. doi: 10.1126/science.163.3870.955

Galán, F., Baker, M. R., Alter, K., and Baker, S. N. (2015). Degraded EEG decoding of wrist movements in absence of kinaesthetic feedback. Hum. Brain Mapp. 36, 643-654. doi: 10.1002/hbm.22653

Ganguly, K., and Carmena, J. M. (2009). Emergence of a stable cortical map for neuroprosthetic control. PLoS Biol. 7:e1000153. doi: 10.1371/journal.pbio.1000153

Gaunt, R. A., Collinger, J. L., Wodlinger, B., Weber, D. J., and Boninger, M. L. (2013). Propioceptive Feedback enables Brain Computer Interface (BCI) Controlled Prosthetic Arm Movement in the Absence of Visual Input. Program No. 374.12. 2013 Neuroscience Meeting Planner. Washington; San Diego: Society for Neuroscience.

Gordon, J., Ghilardi, M. F., and Ghez, C. (1995). Impairments of reaching movements in patients without proprioception. I. Spatial errors. J. Neurophysiol. 73, 347-360.

Hatsopoulos, N. G., Xu, Q., and Amit, Y. (2007). Encoding of movement fragments in the motor cortex. J. Neurosci. 27, 5105-5114. doi: 10.1523/JNEUROSCI.3570-06.2007

Herter, T. M., Korbel, T., and Scott, S. H. (2009). Comparison of neural responses in primary motor cortex to transient and continuous loads during posture. J. Neurophysiol. 101, 150-163. doi: 10.1152/jn.90230.2008

Hochberg, L. R., Bacher, D., Jarosiewicz, B., Masse, N. Y., Simeral, J. D., Vogel, J., et al. (2012). Reach and grasp by people with tetraplegia using a neurally controlled robotic arm. Nature 485, 372-375. doi: 10.1038/nature 11076

Jarosiewicz, B., Chase, S. M., Fraser, G. W., Velliste, M., Kass, R. E., and Schwartz, A. B. (2008). Functional network reorganization during learning in a braincomputer interface paradigm. Proc. Natl. Acad. Sci. U.S.A. 105, 19486-19491. doi: 10.1073/pnas.0808113105

Kalman, R. E. (1960). A new approach to linear filtering and prediction problems. J. Fluids Eng. 82, 35-45. doi: 10.1115/1.3662552

Kuiken, T. A., Miller, L. A., Lipschutz, R. D., Lock, B. A., Stubblefield, K., Marasco, P. D., et al. (2007). Targeted reinnervation for enhanced prosthetic arm function in a woman with a proximal amputation: a case study. Lancet 369, 371-380. doi: 10.1016/S0140-6736(07)60193-7

London, B. M., Jordan, L. R., Jackson, C. R., and Miller, L. E. (2008). Electrical stimulation of the proprioceptive cortex (Area 3a) used to instruct a behaving monkey. IEEE Trans. Neural Syst. Rehabil. Eng. 16, 32-36. doi: 10.1109/TNSRE.2007.907544

Miranda, R. A., Casebeer, W. D., Hein, A. M., Judy, J. W., Krotkov, E. P., Laabs, T. L., et al. (2015). DARPA-funded efforts in the development of novel braincomputer interface technologies. J. Neurosci. Methods 244, 52-67. doi: 10.1016/ j.jneumeth.2014.07.019

Moritz, C. T., Perlmutter, S. I., and Fetz, E. E. (2008). Direct control of paralysed muscles by cortical neurons. Nature 456, 639-642. doi: 10.1038/nature 07418
O’Doherty, J. E., Lebedev, M. A., Ifft, P. J., Zhuang, K. Z., Shokur, S., Bleuler, H., et al. (2011). Active tactile exploration using a brain-machine-brain interface. Nature 479, 228-231. doi: 10.1038/nature10489

Phillis, Y. A. (1985). Controller design of systems with multiplicative noise. IEEE Trans. Automatic Control 30, 1017-1019. doi: 10.1109/TAC.1985. 1103828

Pohlmeyer, E. A., Oby, E. R., Perreault, E. J., Solla, S. A., Kilgore, K. L., Kirsch, R. F., et al. (2009). Toward the restoration of hand use to a paralyzed monkey: brain-controlled functional electrical stimulation of forearm muscles. PLoS ONE 4:e5924. doi: 10.1371/journal.pone.0005924

Pruszynski, J. A., Kurtzer, I., Nashed, J. Y., Omrani, M., Brouwer, B., and Scott, S. H. (2011). Primary motor cortex underlies multi-joint integration for fast feedback control. Nature 478, 387-390. doi: 10.1038/nature 10436

Qian, N., Jiang, Y., Jiang, Z. P., and Mazzoni, P. (2013). Movement duration, Fitts's law, and an infinite-horizon optimal feedback control model for biological motor systems. Neural Comput. 25, 697-724. doi: 10.1162/NECO_ a_00410

Raspopovic, S., Capogrosso, M., Petrini, F. M., Bonizzato, M., Rigosa, J., Di Pino, G., et al. (2014). Restoring natural sensory feedback in real-time bidirectional hand prostheses. Sci. Transl. Med. 6:222ra19. doi: 10.1126/scitranslmed. 3006820

Reilly, K. T., Mercier, C., Schieber, M. H., and Sirigu, A. (2006). Persistent hand motor commands in the amputees' brain. Brain 129, 2211-2223. doi: 10.1093/brain/awl154

Romo, R., Hernandez, A., Zainos, A., and Salinas, E. (1998). Somatosensory discrimination based on cortical microstimulation. Nature 392, 387-390. doi: $10.1038 / 32891$

Sanes, J. N., Mauritz, K. H., Evarts, E. V., Dalakas, M. C., and Chu, A. (1984). Motor deficits in patients with large-fiber sensory neuropathy. Proc. Natl. Acad. Sci. U.S.A. 81, 979-982. doi: 10.1073/pnas.81.3.979

Scott, S. H. (2012). The computational and neural basis of voluntary motor control and planning. Trends Cogn. Sci. 16, 541-549. doi: 10.1016/j.tics.2012. 09.008

Scott, S. H., Cluff, T., Lowrey, C. R., and Takei, T. (2015). Feedback control during voluntary motor actions. Curr. Opin. Neurobiol. 33, 85-94. doi: 10.1016/j.conb.2015.03.006

Sergio, L. E., Hamel-Pâquet, C., and Kalaska, J. F. (2005). Motor cortex neural correlates of output kinematics and kinetics during isometric-force and armreaching tasks. J. Neurophysiol. 94, 2353-2378. doi: 10.1152/jn.00989.2004

Shadmehr, R., and Krakauer, J. W. (2008). A computational neuroanatomy for motor control. Exp. Brain Res. 185, 359-381. doi: 10.1007/s00221-008-1280-5

Shadmehr, R., and Mussa-Ivaldi, S. (2012). Biological Learning and Control: How the Brain Builds Representations, Predicts Events, and Makes Decisions. Cambridge, MA: The MIT Press. doi: 10.7551/mitpress/9780262016964.001. 0001

Shenoy, K. V., Kaufman, M. T., Sahani, M., and Churchland, M. M. (2011). A dynamical systems view of motor preparation: implications for neural prosthetic system design. Prog. Brain Res. 192, 33-58. doi: 10.1016/B978-0-44453355-5.00003-8

Shenoy, K. V., Sahani, M., and Churchland, M. M. (2013). Cortical control of arm movements: a dynamical systems perspective. Annu. Rev. Neurosci. 36, 337-359. doi: 10.1146/annurev-neuro-062111-150509

Shenoy, K. V., and Carmena, J. M. (2014). Combining decoder design and neural adaptation in brain-machine interfaces. Neuron 84, 665-680. doi: 10.1016/j.neuron.2014.08.038

Suminski, A. J., Tkach, D. C., Fagg, A. H., and Hatsopoulos, N. G. (2010). Incorporating feedback from multiple sensory modalities enhances brain-machine interface control. J. Neurosci. 30, 16777-16787. doi: 10.1523/JNEUROSCI.3967-10.2010

Taylor, D. M., Tillery, S. I., and Schwartz, A. B. (2002). Direct cortical control of 3D neuroprosthetic devices. Science 296, 1829-1832. doi: 10.1126/science. 1070291

Todorov, E., and Jordan, M. I. (2002). Optimal feedback control as a theory of motor coordination. Nat. Neurosci. 5, 1226-1235. doi: 10.1038/ nn963 
Velliste, M., Perel, S., Spalding, M. C., Whitford, A. S., and Schwartz, A. B. (2008). Cortical control of a prosthetic arm for self-feeding. Nature 453, 1098-1101. doi: 10.1038/nature 06996

Wolpert, D. M., and Miall, R. C. (1996). Forward models for physiological motor control. Neural Netw. 9, 1265-1279. doi: 10.1016/S0893-6080(96) 00035-4

Wolpert, D. M., Miall, R. C., and Kawato, M. (1998). Internal models in the cerebellum. Trends Cogn. Sci. 2, 338-347. doi: 10.1016/S1364-6613(98) $01221-2$
Conflict of Interest Statement: The authors declare that the research was conducted in the absence of any commercial or financial relationships that could be construed as a potential conflict of interest.

Copyright (C) 2015 Galán and Baker. This is an open-access article distributed under the terms of the Creative Commons Attribution License (CC BY). The use, distribution or reproduction in other forums is permitted, provided the original author(s) or licensor are credited and that the original publication in this journal is cited, in accordance with accepted academic practice. No use, distribution or reproduction is permitted which does not comply with these terms. 